\title{
Microstructure inhomogeneity of centrifugally cast ductile iron pipes and its effect on mechanical properties
}

\author{
A. Wassilkowska* \\ Faculty of Environmental Engineering, Cracow University of Technology, ul. Warszawska 24, PL-31155 Krakow, Poland
}

Received 26 November 2015, received in revised form 15 June 2016, accepted 1 March 2017

\begin{abstract}
The microstructure of two centrifugally cast ductile iron pipes with a diameter of DN100 and wall thickness of $6 \mathrm{~mm}$ was studied. After casting, the pipes undergo annealing and finishing with anticorrosion coatings. Tensile tests were performed using longitudinal samples, cut in an appropriate manner from the pipe wall. Depending on the graphite morphology and the concentration area of casting defects, tensile samples cut from the same ductile iron pipe may show significant scatter of mechanical parameters. Thus, when tensile samples are machined from pipe ready for installation, the results of mechanical tests must be carefully interpreted. Particular attention should be paid to the porosity, present in the cross-section of the strained samples. The defective material has insufficient strength and very low elongation, i.e. tensile properties of the individual sample could be well below the required minimum of $420 \mathrm{MPa}$ and $10 \%$ in the standard EN 545.
\end{abstract}

K e y w or d s: ductile iron, water pipes, centrifugal casting, tensile properties, internal porosity, chunky graphite

\section{Introduction}

Ductile iron pipes produced by centrifugal casting are used, for example, like water and gravity sewer pipelines $[1,2]$. The typical dimensions of centrifugally cast pipes are the length of $5-6 \mathrm{~m}$ and diameter of $80-700 \mathrm{~mm}$, but larger diameters are also produced according to standard (1200 and $2000 \mathrm{~mm}$ ). The pipe casting takes place in a rotating horizontal mould from heat resistant steel, wherein the mould wall is cooled with water. The advantage of centrifugal casting is the precise control of pipe thickness, where the minimum thickness of the ductile iron pipe is determined by its class of pressure [1]. For water pipes, a commonly used class K9 is an optimum compromise between the strength of pipeline against internal pressure, resistance to longitudinal deflection (especially for small pipe diameters) and stiffness (particularly for large diameters) [3]. The current standard EN 545:2010 requirements include new classes C40-C100, calculated from the wall thickness, which open up the possibility of thinning the ductile iron water pipes [3]. Presently one can find on the market pipes, having thickness even less than $3 \mathrm{~mm}$. However, reducing a wall thickness changes the crystallisation conditions during centrifugal casting, and additional annealing of pipes is required to obtain a proper structure in their cross-section and length. The strength properties of water pipes, i.e. deformation plasticity of min. $10 \%$, yield strength of min. $270 \mathrm{MPa}$ and tensile strength of min. $420 \mathrm{MPa}$ [1], clearly point to the ferritic grade of ductile iron after casting and annealing, e.g. EN-GJS-450-10 [4]. Production quality control of ductile iron pipes includes uniaxial tensile tests of ingot samples [1]. The aim of this study was to determine the microstructure characteristics in the cross-section of the pipe wall. Mechanical properties of ductile iron taken from the pipe ready for installation have also been verified.

\section{Experimental procedure}

Two brand-new ductile iron pipes from different manufacturers were used for this study, both with a nominal diameter of DN100 and almost equal wall

*Corresponding author: e-mail address: awassilkowska@pk.edu.pl 

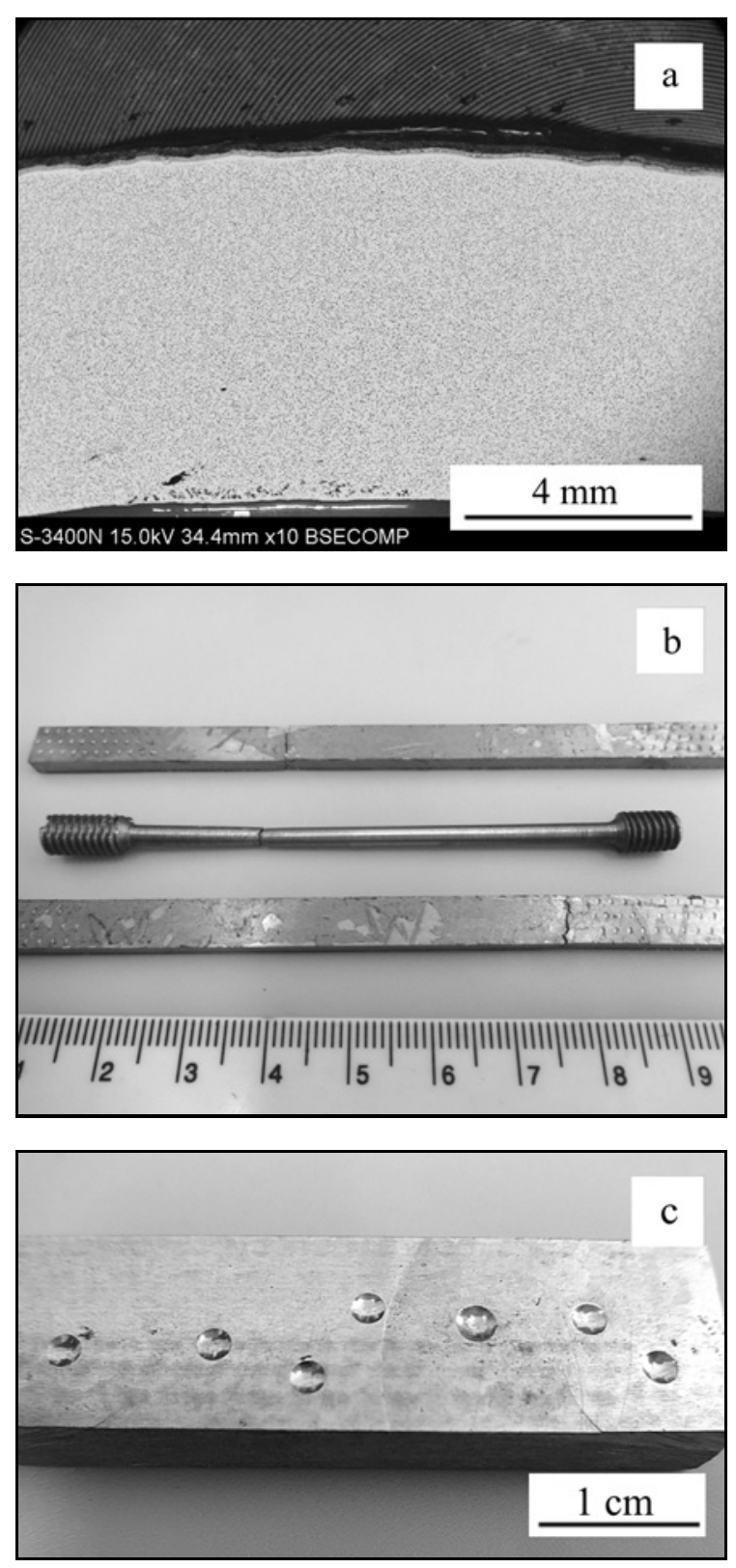

Fig. 1. Experimental samples: (a) cross-section microstructure of the pipe wall; (b) two kinds of samples for uniaxial tensile tests; (c) the Brinell hardness measurement.

thickness $(6.3 \mathrm{~mm}$ for pipe $\mathrm{R} 1$ and $6.2 \mathrm{~mm}$ for pipe $\mathrm{R} 2$ ). The pipes have anticorrosion coatings from the outside and from the inside, which in mechanical studies are removed from the specimens. For microstructure analysis, transverse metallographic specimens were prepared, and tensile specimens were machined from the pipe wall (Fig. 1).

The Brinell hardness was measured on planeparallel samples using a steel ball indenter with a diameter of $5 \mathrm{~mm}$ at a pressure of $7.335 \mathrm{kN}$ (Fig. 1c). Uniaxial tensile properties were examined for different longitudinal samples (Fig. 1b) according to EN ISO
6892-1 [5]. Samples of diameter $\varnothing 3 \mathrm{~mm}$ had threaded handle made from an entire pipe thickness and were strained on Zwick/Roell machine using an extensometer of $50 \mathrm{~mm}$ gauge length. Samples with a flat section and proportionality of $k=11.3$ were tested on INSTRON machine at a traverse speed of $1 \mathrm{~mm} \mathrm{~min}^{-1}$ and base $50 \mathrm{~mm}$ for strain measuring. The effect of microstructure inhomogeneity across the pipe wall on the strength parameters was examined using two series of flat samples. Samples machined with a wider side near the outer surface of the pipe made Batch I (External). Samples thinned so that their wider side adhered to the inner surface of the pipe made Batch II (Internal). The microstructure analysis of ductile iron pipes was performed in the wall cross-section (Fig. 1a) using a scanning electron microscope Hitachi S-3400N equipped with an energy dispersive X-ray detector (EDS) from ThermoScientific ${ }^{\circledR}$.

\section{Results and discussion}

Figures 2 and 3 show the microstructure in the wall cross-section of the ductile iron pipes tested. Figures $2 \mathrm{a}$ and $3 \mathrm{a}$ illustrate the microstructure near the outer surface of the pipes. Even at a low image magnification of $50 \times$ and $200 \times$, the top layer of the protective paint filling pores and a roughness of underneath zinc layer can be seen. The protective zinc coating is applied by thermal spraying on the oxidised surface of the annealed pipes. According to the standard [1], the iron oxides do not need to be removed during the preparation of the surface for metallising. The thickness of oxide layer is about $80-100 \mu \mathrm{m}$. Even a decarburised layer (ferrite without graphite particles) having a thickness of approximate $50-60 \mu \mathrm{m}$ can be seen on the entire outer surface of the pipes. This layer is formed as a result of heating the centrifugally cast pipes for annealing to above $950^{\circ} \mathrm{C}$. Under the decarburised layer, there is an area with a smaller volume fraction of graphite and next - practically uniform distribution of graphite in a ferritic matrix.

Figures $2 \mathrm{~b}$ and $3 \mathrm{~b}$ show the microstructure of the tested pipes in the middle-thickness of the pipe wall. The shape and size of graphite particles were determined at a standard magnification of $100 \times$ (no etched samples). When the internal wall thickness of centrifugally cast pipes, i.e. the pipe core, was being cooled down slowly, local changes of the graphite morphology and shrinkage cavities were observed. In a test sample R1 (Fig. 2c), surface irregularities were recorded, accompanied by an area of flake graphite and once in a while - by slight microporosity. In contrast, the internal surface of a test sample R2 was relatively smooth (Fig. 3c), but there was a regular band of large subsurface pores present. The cavities reached to a depth of about $0.3 \mathrm{~mm}$, so in the specimen cross-section the 

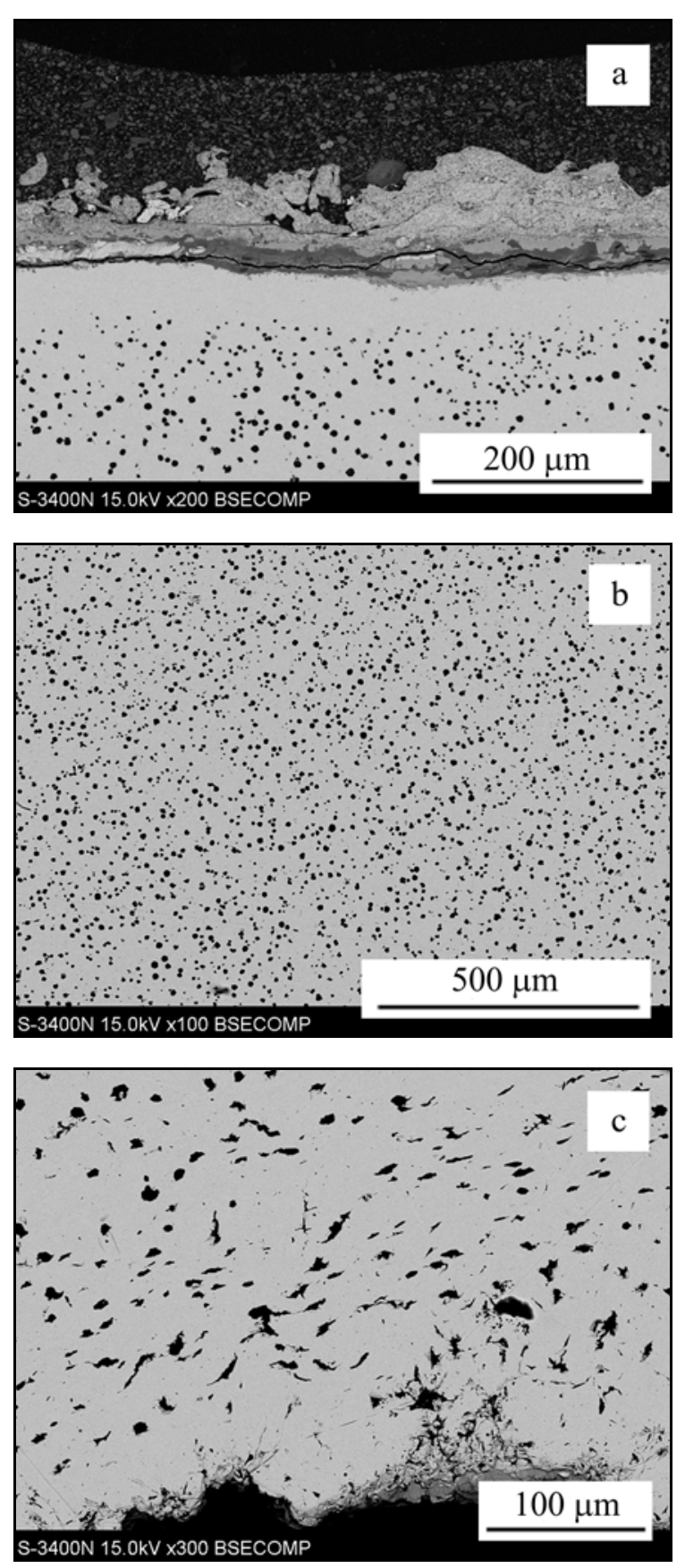

Fig. 2. The microstructure of pipe R1: (a) the outer surface of the pipe with anticorrosion coating; (b) the ductile iron structure in the middle of the pipe wall; (c) near internal pipe surface.

porosity was visible with the naked eye. From the same side of the sample, i.e. at the inner surface of the pipe, the Brinell hardness obtained was 158 to $164 \mathrm{HBW}$ for both pipes, and therefore, no effect of pores was registered.

According to the standard [1], the hardness of ductile iron pipes cannot exceed $230 \mathrm{HBW}$, which depends mainly on the type of the matrix metal. The iron
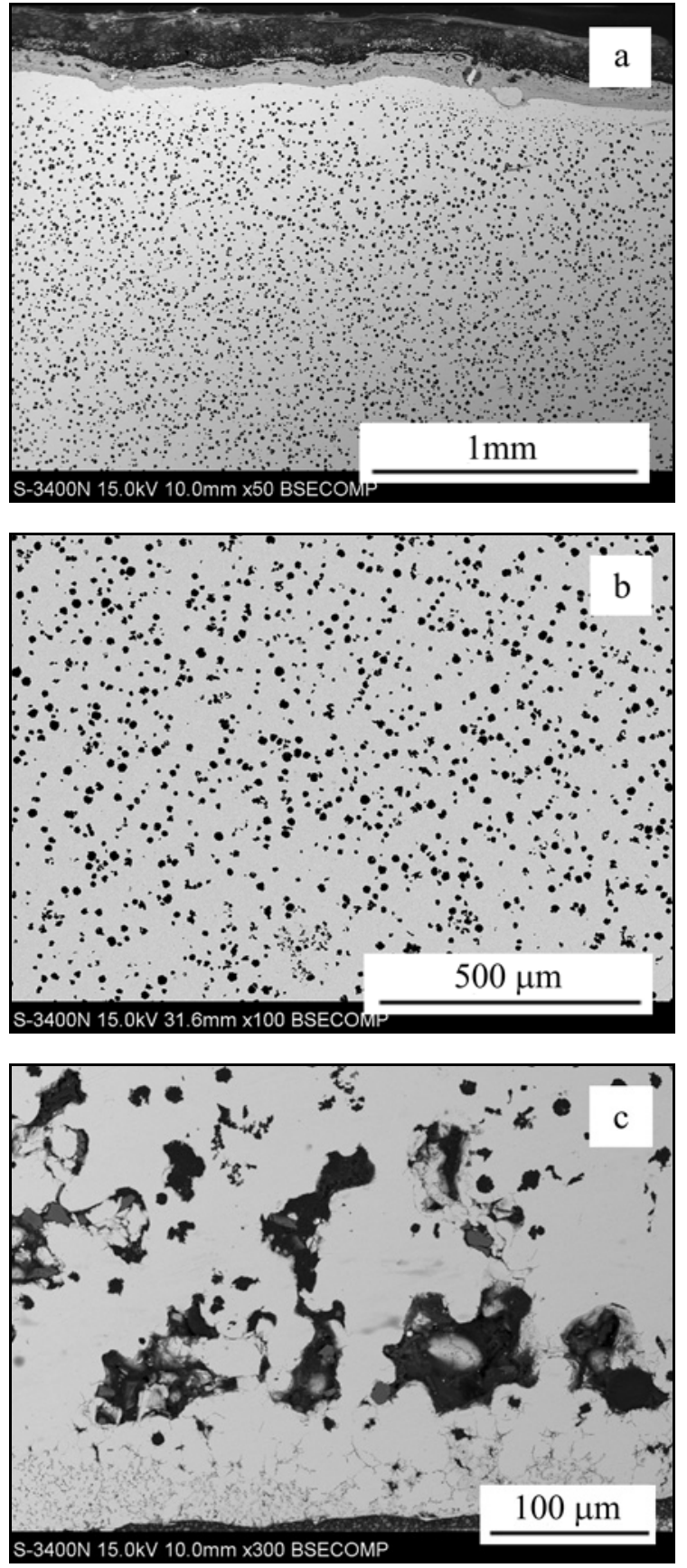

Fig. 3. The microstructure of pipe R2: (a) the outer surface of the pipe with anticorrosion coating; (b) the ductile iron structure in the middle of the pipe wall; (c) shrinkage porosity visible near internal pipe surface.

matrix after annealing is ferritic in both pipes under study, so there are small differences in the measured values.

When machining longitudinal tensile specimens of $\varnothing 3 \mathrm{~mm}$ in wall cross-section, the protective coatings on the two surfaces of the pipe are removed, as well as the decarburised layer and the layer containing pores. As a result, the samples are made from the inside volume 

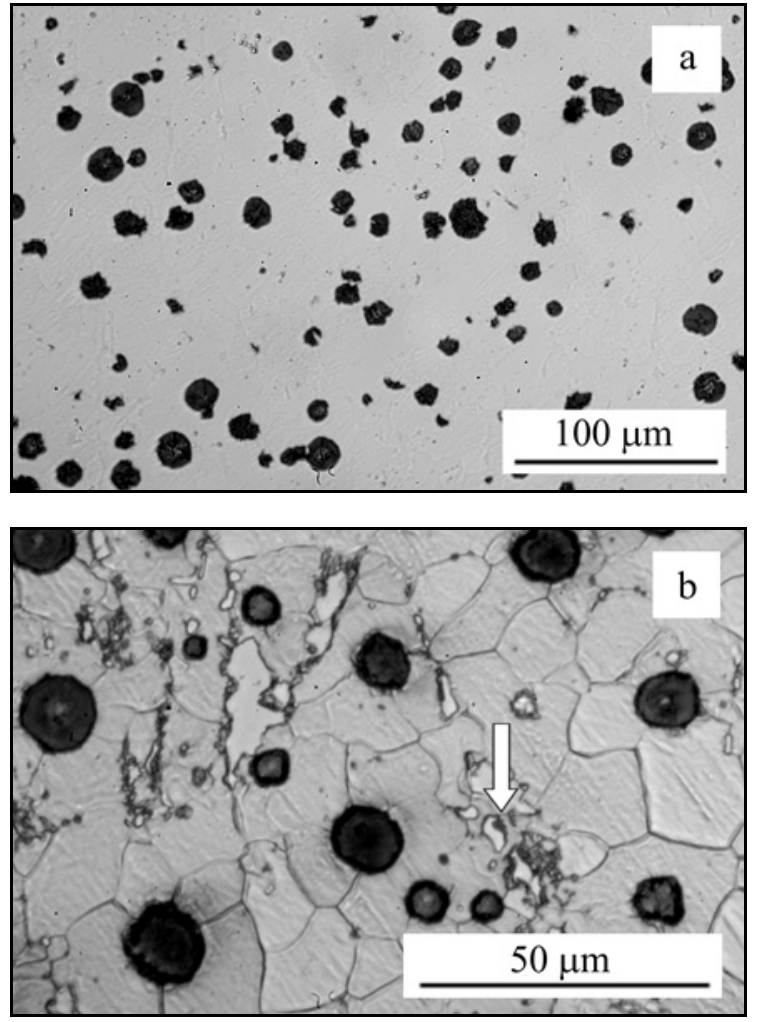

Fig. 4. The microstructure of specimen R1: (a) non-etched specimen; (b) specimen etched in Nital solution; nonmetallic inclusions shown with an arrow.

of the ductile iron wall and exclude those structures. Careful study of the distribution and size of graphite particles at a higher magnification showed that the samples of ductile iron pipes under study have different microstructure (Figs. 4 and 5).

While the graphite particles are uniformly distributed in the ferritic matrix and have a similar diameter of $10 \mu \mathrm{m}$ (determined according to [6]), the presence of nonmetallic precipitates was observed in both materials under study. In specimen R1 there are precipitates with irregular shape, visible after etching of metallographic specimen, disclosed between ferritic grains (Fig. 4b). In specimen R2, the nonmetallic particles, with a shape similar to that of graphite, were observed as dark inclusions, without etching of metallographic sample. Moreover, the shape of the graphite particles in specimen $\mathrm{R} 2$ is not perfectly spherical, as would be expected after completing the spheroidization process.

The greatest change in the microstructure in the cross-section of the pipe wall was observed near the internal surface of pipe R2 (Fig. 6). While sample R1 shows spheroidal graphite through the pipe wall, and there is a lack of shrinkage porosity and other casting defects, in specimen R2 significant micro shrinkages associated with the solidification of iron are observed,
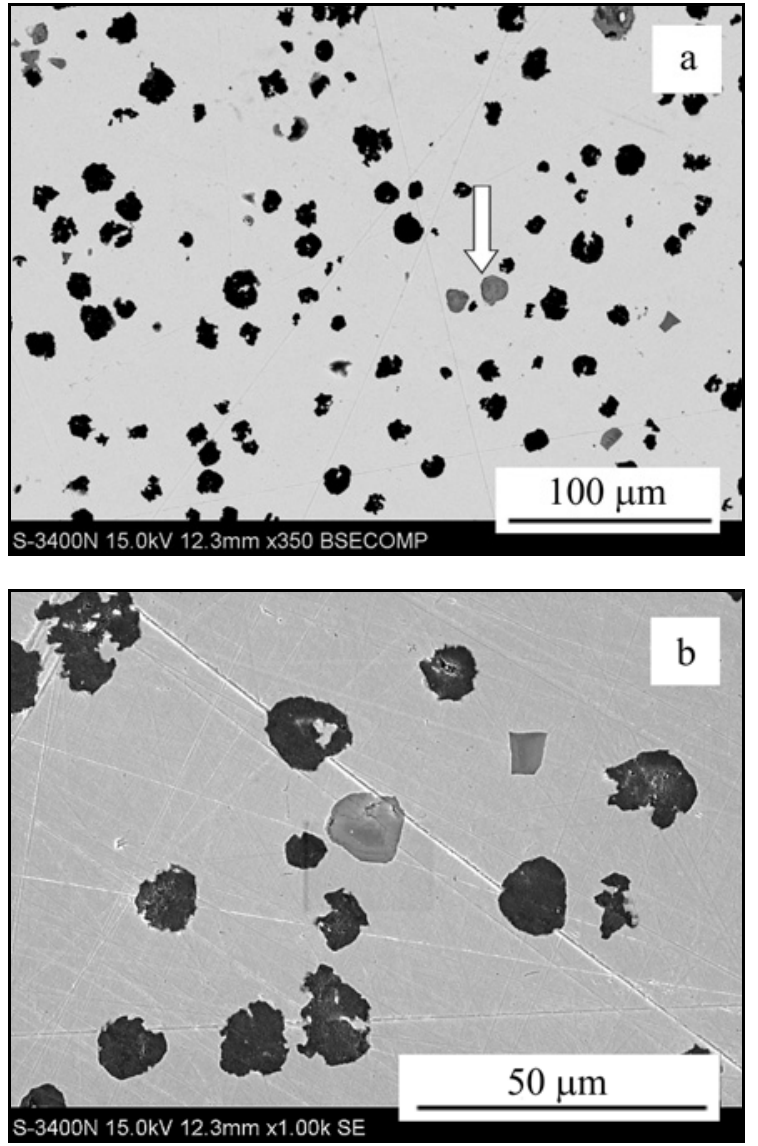

Fig. 5. The microstructure of specimen R2: (a) nonmetallic particles in a ferritic matrix - shown with an arrow; (b) particles morphology at higher magnification.

and chunky graphite is formed. Micro shrinkages are formed primarily due to metal shrinkage during solidification in a wide temperature range, as well as due to gas release from the mass of the core when lowering the temperature.

The shrinkage porosity areas in the microstructure of the ductile iron solidify last. This indirectly indicates that probably the temperature of the melt, before pouring, was higher than necessary, which leads to the dissolution of more gases into the melted alloy and larger temperature gradient between the alloy and the casting mould. This microstructure means significantly lower mechanical properties (tensile strength and elongation) of the tested material from this part of ductile iron pipes.

The results of the tensile tests for both pipes examined are compared in Table 1. Test specimen R1 had exceptionally good mechanical properties. The elongation values were in the range of $13-15 \%$ and in all samples tested were greater than required in the standard [1]. The same cannot be said for the relative elongation of sample R2, although the structure in the middle of the wall in the materials $\mathrm{R} 1$ and $\mathrm{R} 2$ was al- 

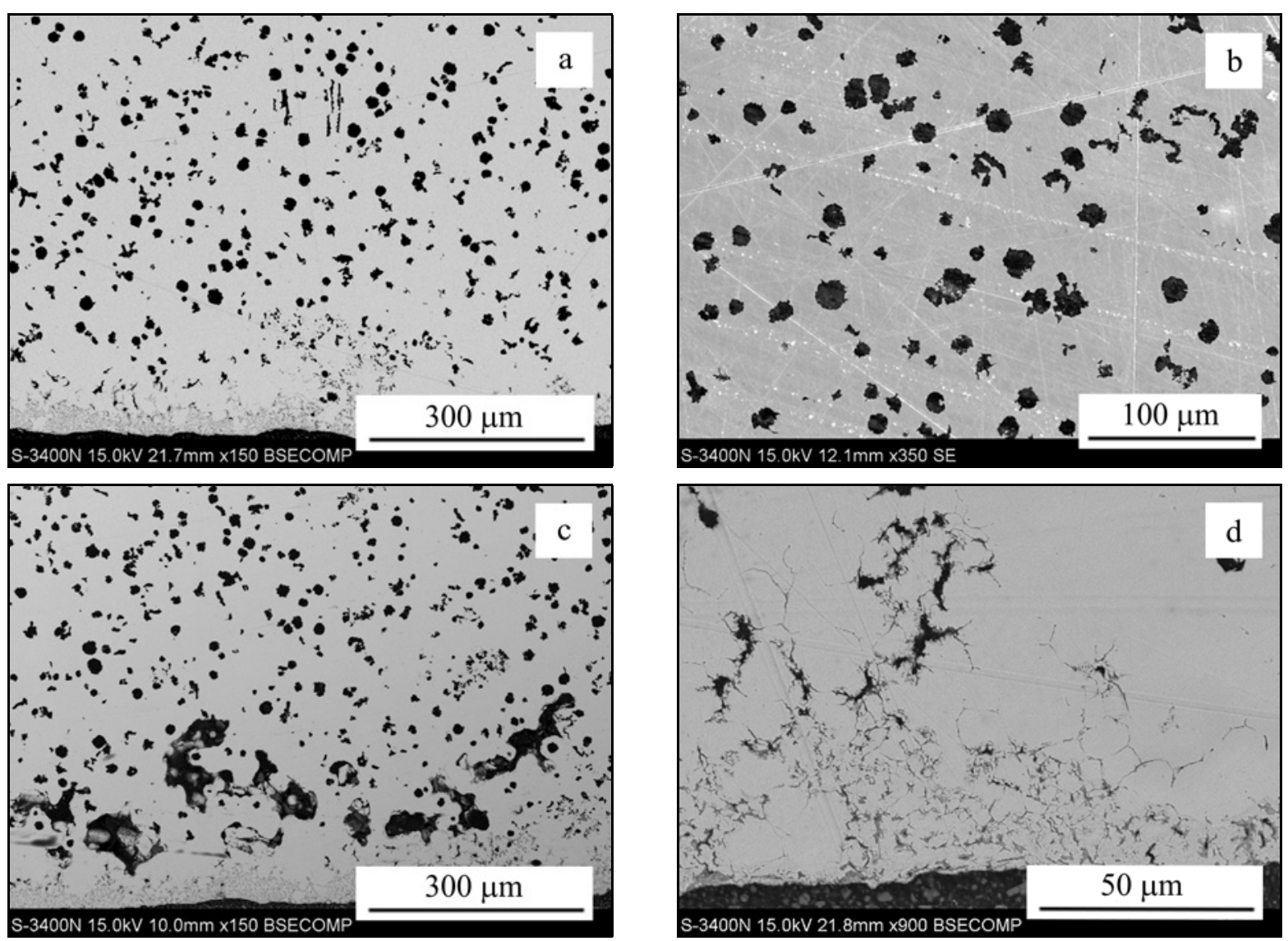

Fig. 6. Cross-section of the wall of ductile iron pipe R2: $(\mathrm{a}-\mathrm{d})$ the microstructure near the internal surface of the pipe.

Table 1. Tensile test results of round samples from pipes $\mathrm{R} 1$ and $\mathrm{R} 2\left(R_{\mathrm{m}}\right.$ is tensile strength, $R_{0.2}$ is yield strength, and $A$ is total elongation)

\begin{tabular}{cccccccc}
\hline & \multicolumn{3}{c}{ Pipe R1 } & & \multicolumn{3}{c}{ Pipe R2 } \\
\cline { 2 - 3 } \cline { 7 - 8 } No. & $R_{\mathrm{m}}(\mathrm{MPa})$ & $R_{\mathrm{p} 0.2}(\mathrm{MPa})$ & $A(\%)$ & & $R_{\mathrm{m}}(\mathrm{MPa})$ & $R_{\mathrm{p} 0.2}(\mathrm{MPa})$ & $A(\%)$ \\
\hline 1 & 463 & 318 & 15.0 & & 458 & 308 & 13.5 \\
2 & 446 & 310 & 12.9 & & 453 & 324 & 6.7 \\
3 & 461 & 318 & 14.1 & & 468 & 320 & 13.8 \\
4 & - & - & - & & 451 & 350 & 9.7 \\
\hline
\end{tabular}

Ta b le 2. Tensile test results of flat samples from batches I and II (Pipe R2) ( $R_{\mathrm{m}}$ is tensile strength, $R_{0.2}$ is yield strength, and $A$ is total elongation)

\begin{tabular}{cccccccc}
\hline & \multicolumn{3}{c}{ Batch I (External) } & & \multicolumn{3}{c}{ Batch II (Internal) } \\
\cline { 2 - 3 } No. & $R_{\mathrm{m}}(\mathrm{MPa})$ & $R_{\mathrm{p} 0.2}(\mathrm{MPa})$ & $A(\%)$ & & $R_{\mathrm{m}}(\mathrm{MPa})$ & $R_{\mathrm{p} 0.2}(\mathrm{MPa})$ & $A(\%)$ \\
\hline 1 & 467 & 314 & 13.8 & & 400 & 301 & 3.4 \\
2 & 464 & 313 & 11.0 & & 432 & 301 & 6.2 \\
3 & 467 & 313 & 14.6 & & 424 & 301 & 4.2 \\
\hline
\end{tabular}

most identical. The values of elongation varied widely, and for sample no. 2 was significantly less than $10 \%$. The spread in elongation values indicates that poros- ity near the internal surface of the pipe (Fig. 3b) has not been completely removed in all tensile specimens. The effect of heterogeneous microstructure in the wall 


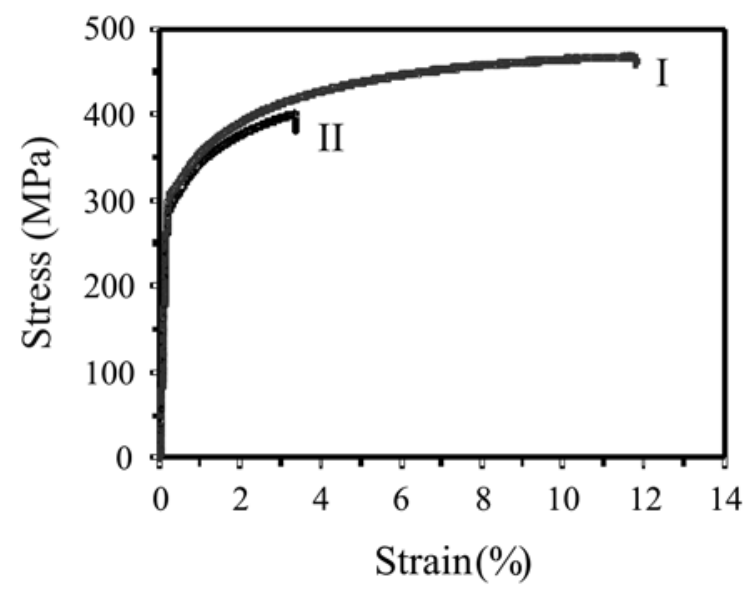

Fig. 7. Tensile curves of specimens No. 1 from batches I and II (Pipe R2).

cross-section of centrifugally cast ductile iron pipe on the tensile parameters has been reflected to the proportional flat specimens from pipe R2. The results of two series of tests are shown in Table 2.

The tensile results in Tables 1 and 2 show that Batch I (External) is characterised by high and stable strength parameters and the values are similar to those obtained for the round samples of pipe R1. The lowest strength parameters were obtained for Batch II (Internal), wherein samples to be stretched were cut near the internal surface of the pipe. In this case, the minimum elongation of $10 \%$ was not reached, and one of the tensile samples (no. 1) showed strength below the required minimum of $420 \mathrm{MPa}$, according to EN 545 [1]. The overall effect of the observed defects in ductile iron microstructure was less pronounced in the strength but had a bigger impact on the material elongation. Similar results from the study of chunky graphite and micro shrinkages, but on fatigue strength of ductile iron, are obtained in work [7]. Figure 7 presents the tensile curves of samples no. 1 from Batches I and II, demonstrating differences in their tensile properties.

\section{Conclusion}

Porosity and the emergence of chunky graphite, which developed near the internal surface of centrifugally cast ductile iron pipes, may be considered as dangerous defects for water pipes under pressure. Microstructure inhomogeneity causes instability in the strength parameters, which is manifested mainly in the decline of relative specimen elongation under a standardised minimum of $10 \%$. The reduced working cross-section of ductile iron pipes, caused by shrinkage porosity and chunking graphite, is a negative factor for their lifespan.

\section{Acknowledgement}

The study was made possible with the financial support of Municipal Water Supply and Sewage S.A., Cracow, Poland (Project no. S-3/412/2012).

\section{References}

[1] EN 545:2010: Ductile Iron Pipes, Fittings, Accessories and their Joints for Water Pipelines - Requirements and Test Methods.

[2] EN 598:2007: Ductile Iron Pipes, Fittings, Accessories and their Joints for Sewerage Applications - Requirements and Test Methods.

[3] Rammelsberg, J.: The Annual Journal of the EADIPS, 45, 2011, p. 19.

[4] EN 1563:2011: Founding - Spheroidal graphite cast irons.

[5] EN ISO 6892-1:2009: Metallic Materials -Tensile Testing - Part 1: Method of the Test at Room Temperature.

[6] ISO 945-1:2008: Microstructure of Cast Irons - Part 1: Graphite Classification by Visual Analysis.

[7] Ferro, P., Lazzarin, P., Berto, F.: Mater. Sci. Eng. A, 544, 2012, p. 122. doi:10.106/i.msea.2012.06.024 